\title{
Reconfigurable Circularly Polarized Cross-Dipole Antenna with a Near-Field Resonant Parasitic Element
}

\author{
${ }^{1}$ Son Xuat Ta, ${ }^{2}$ Ikmo Park, ${ }^{3,4}$ Richard W. Ziolkowski \\ ${ }^{1}$ School of Electronics and Telecommunications, Hanoi University of Science and Technology, Hanoi, Vietnam \\ e-mail: tasonxuat@yahoo.com \\ ${ }^{2}$ Department of Electrical and Computer Engineering, Ajou University, Republic of Korea \\ e-mail: ipark@ajou.ac.kr \\ ${ }^{3}$ Global Big Data Technologies Centre, University of Technology Sydney \\ ${ }^{4}$ Department of Electrical and Computer Engineering, University of Arizona, AZ, USA \\ e-mail: ziolkowski@ece.arizona.edu
}

\begin{abstract}
This paper presents a polarization switchable circularly polarized (CP) antenna that consists of a compact single-feed crossed dipole loaded with a near-field resonant parasitic (NFRP) element. This antenna is placed above a metallic reflector to enhance its gain and broadside radiation pattern. A meander line with an arrowhead-shaped ending was applied to all arms of the crossed dipole and NFRP elements for compactness. By adjusting the end size of the NFRP element, the antenna was capable of switching its polarization from right-hand circular polarization to left-hand circular polarization and vice versa. The reconfigurability is realized by controlling the ON/OFF states of 8 PIN-diodes, which are inserted into the arrowhead endings of the NFRP element. For the RHCP mode, the antenna yields a $\left|S_{11}\right|<-10 \mathrm{~dB}$ bandwidth of 1.465-1.665 GHz and a 3-dB axial ratio (AR) bandwidth of 1.535-1.590 GHz. For the LHCP mode, the antenna yields a $\left|S_{11}\right|<-10 \mathrm{~dB}$ bandwidth of 1.50-1.69 GHz and a 3-dB AR bandwidth of 1.545-1.585 GHz. Moreover, in both modes, the antenna yields a good broadside radiation pattern with a gain of $>6.0 \mathrm{dBic}$ and a radiation efficiency of $>70 \%$ across its operational bandwidth.
\end{abstract}

Index Terms - circular polarization, crossed dipole, near-field resonant parasitic element, switchable polarization.

\section{INTRODUCTION}

Polarization reconfigurable circularly polarized (CP) antennas are able to generate both left-hand CP (LHCP) and right-hand CP (RHCP). These antennas can be used for avoiding multipath effects, for polarization coding, and for enhancing system capacity. Different $\mathrm{CP}$ antenna designs have been developed to achieve reconfigurable polarization, including patch antennas [1], [2], aperture-coupled patch antennas [3], [4], crossed-dipole antennas [5], sequentially rotated array antennas [6], loop antennas [7], and metasurface-based antennas [8], [9]. In addition, there are several methods that can be used to achieve reconfigurable $\mathrm{CP}$ radiation, such as reconfigurable primary radiating elements [1], [2], [7], reconfigurable feeding structures [3]-
[6], [10], the reconfigurable slotted ground plane [3], physically rotating the metasurface around the center with respect to the antenna [8], and using the active metasurface [9].

A variety of antenna types have been engineered with metamaterials (MTMs) or MTM-inspired structures to enhance their capability or to achieve novel functions [10]. A typical example of this class is the near-field resonant parasitic (NFRP) antenna, which is conventionally constructed from a single MTM unit cell and incorporated with an electrically small driven element. A single MTM unit cell can be easily incorporated with electronic switching components to change its properties, and consequently, the NFRP antennas have good potential to realize reconfigurable characteristics [11], [12].

In this paper, we investigated the polarization reconfigurability of a single-feed crossed-dipole antenna loaded with an NFRP element. The CP reconfigurability (switching the polarization from RHCP to LHCP and vice versa) was obtained by adjusting the end sizes of the individual NFRP arms. The adjustment was electronically realized by controlling the state of the PIN diodes, which were inserted into the arrowhead endings of the NFRP element. The proposed design had a compact size, a broad impedance matching bandwidth, a good broadside CP radiation, and a high radiation efficiency (RE). The antenna was characterized using the ANSYS high-frequency structure simulator (HFSS).

\section{ANTENNA GEOMETRY AND CHARACTERISTICS}

Figure 1 shows the geometry of a crossed-dipole antenna loaded with a NFRP element [13], [14]. The antenna was placed above a $150 \mathrm{~mm} \times 150 \mathrm{~mm}$ ground plane at a distance of $H_{a}$. Its primary radiating elements were printed on two substrates made of Rogers RT/Duroid 5880 material $\left(\varepsilon_{r}=2.2\right.$ and $\tan \delta=0.0009$ ) with a thickness of $0.508 \mathrm{~mm}$. The 
substrates were stacked together with no air gap. The crossed dipole was printed on both sides of substrate 1 , whereas the NFRP element was printed on the top side of substrate 2 . The crossed dipole was incorporated with a pair of vacant-quarter printed rings to produce $\mathrm{CP}$ radiation and to match to the 50$\Omega$ coaxial line without a matching network [15]. To obtain a compact size, meander lines with an arrowhead-shaped trace at the end were employed in both the crossed dipole and the

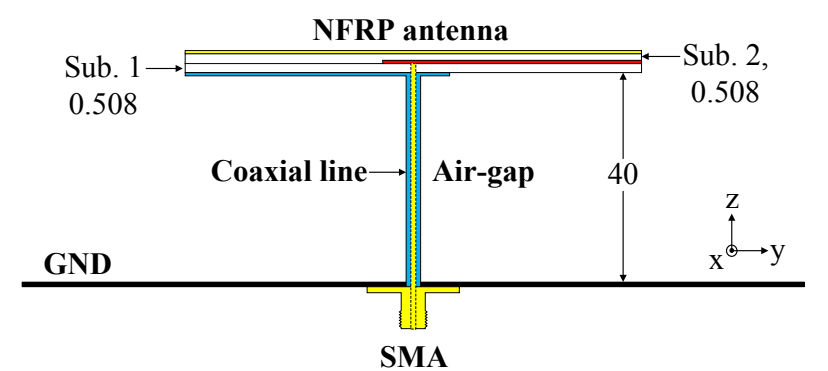

(a)

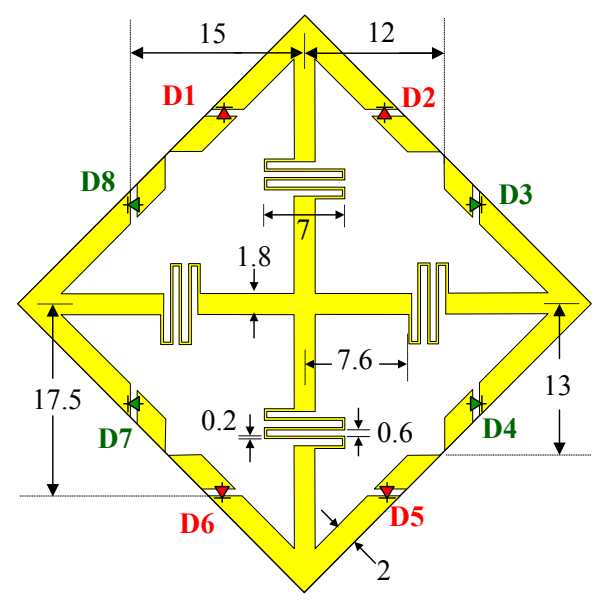

(b)

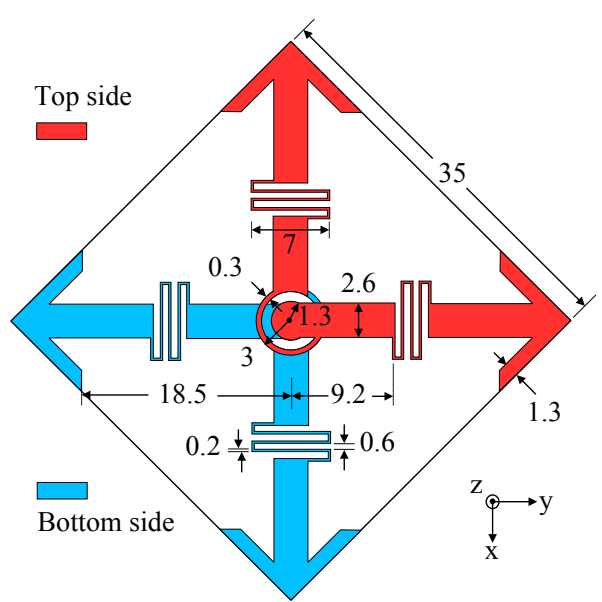

(c)

Fig.1. Geometry of the reported antenna (units in $\mathrm{mm}$ ): (a) crosssectional view, (b) top view of reconfigurable NFRP element, and (c) top view of crossed-dipole element.
NFRP elements. Eight PIN diodes (D1 D8) were inserted into the arrowhead ends of the NFRP element. The reconfigurability was electronically realized by controlling the ON/OFF states of each group of PIN diodes; i.e., when the red diodes (D1, D2, D5, and D6) were ON and the green diodes (D3, D4, D7, and D8) were OFF, the antenna radiated an RHCP mode. In the opposite state of the diodes, when the red diodes (D1, D2, D5, and D6) were OFF and the green

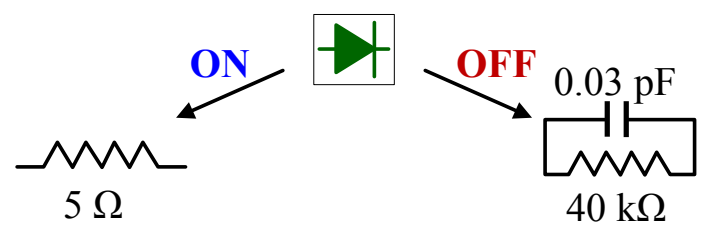

Fig. 2. Linear circuit model of the PIN diode in both the ON and OFF states used in the simulations.

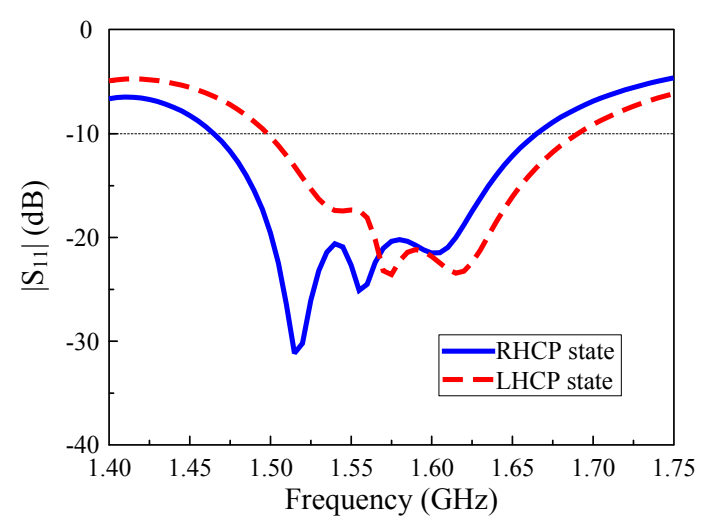

(a)

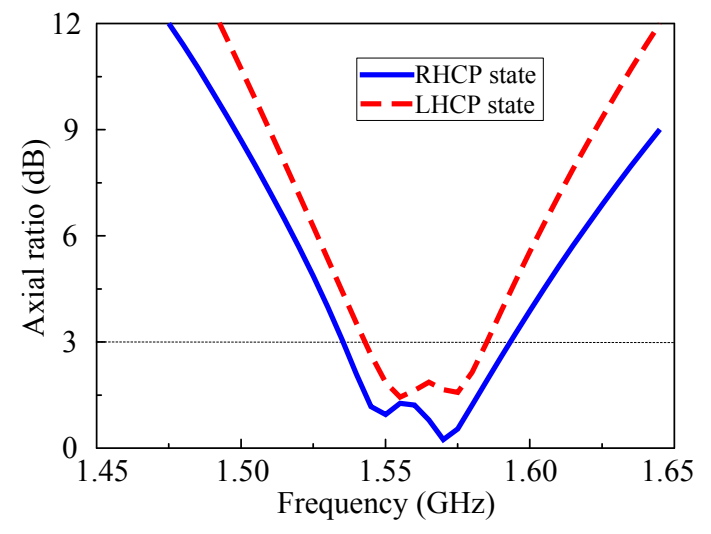

(b)

Fig. 3. Simulated (a) $\left|\mathrm{S}_{11}\right|$ and (b) AR values of the reconfigurable CP antenna for the RHCP and LHCP states.

diodes (D3, D4, D7, and D8) were ON, the antenna radiated an LHCP mode.

To investigate the reconfigurable polarization of the antenna computationally, an electrical model of the 
MA4SPS402 PIN diode from M/A-Com [16] was integrated into the HFSS model. Based on information extracted from the data sheet, an equivalent linear circuit model of the diode is shown in Fig. 2. It has a $5 \Omega$ forward resistance in the $\mathrm{ON}$ state, whereas it has an equivalent parallel circuit of $\mathrm{C}=0.03$ $\mathrm{pF}$ and $\mathrm{R}=40 \mathrm{k} \Omega$ in the OFF state.

Figure 3 shows the simulated $\left|S_{11}\right|$ and $A R$ values of the reconfigurable antenna for both states. It can be seen that for both states, the antenna yielded a broadband characteristic in terms of impedance matching and $\mathrm{CP}$ radiation. For the RHCP state, the antenna yielded a $\left|\mathrm{S}_{11}\right|<-10 \mathrm{~dB}$ bandwidth of 1.465 to $1.665 \mathrm{GHz}(200 \mathrm{MHz})$ and a 3-dB AR bandwidth of 1.535 to $1.590 \mathrm{GHz}(55 \mathrm{MHz})$. For the LHCP state, the impedance matching and 3-dB AR bandwidths were slightly narrower than those of the RHCP state; its $\left|\mathrm{S}_{11}\right|<-10 \mathrm{~dB}$ bandwidth was 1.50 to $1.69 \mathrm{GHz}(190 \mathrm{MHz})$, and its $3-\mathrm{dB}$ AR bandwidth was 1.545 to $1.585 \mathrm{GHz}(40 \mathrm{MHz})$.

Figure 4 shows the simulated $1.56-\mathrm{GHz}$ radiation
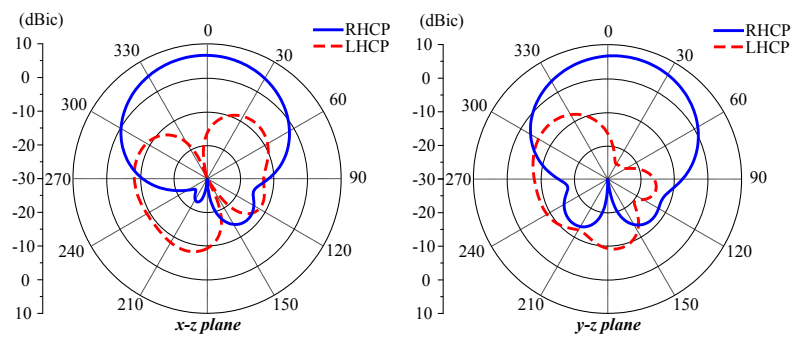

(a)
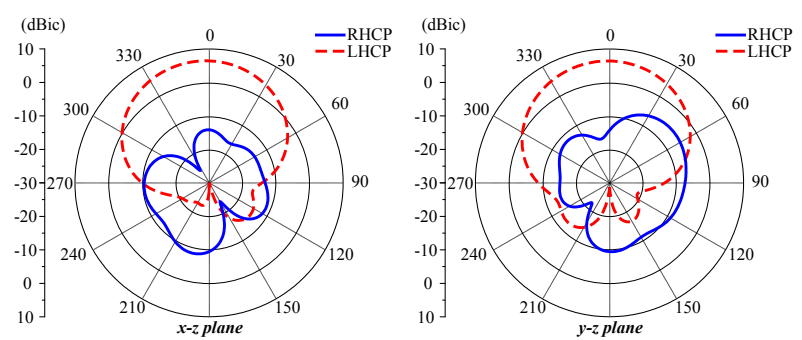

(b)

Fig. 4. Simulated radiation pattern of the reconfigurable $\mathrm{CP}$ antenna at 1.56 GHz: (a) RHCP and (b) LHCP states.

patterns of the reconfigurable antenna for the RHCP and LHCP states. In both states, the antenna yielded good broadside radiation patterns with a symmetric profile and high front-to-back (F-B) ratio. The antenna in an RHCP state resulted in a gain of $7.6 \mathrm{dBic}$, a F-B ratio of $15.9 \mathrm{~dB}$, and half-power beamwidths (HPBWs) of 76 and 73 degrees in the $x-z$ and $y-z$ planes, respectively. The antenna in the LHCP mode resulted in a gain of $6.5 \mathrm{dBic}$, a F-B ratio of 16 $\mathrm{dB}$, and HPBWs of 72 and 76 degrees in the $x-z$ and $y-z$ planes, respectively. Additionally, in both states, the HFSS simulations resulted in an RE of $>70 \%$ across the operational bandwidth.

\section{CONCLUSION}

In this study, we have presented a polarization reconfigurable CP antenna, which is composed of a singlefeed crossed dipole antenna loaded with a reconfigurable NFRP element. By adjusting the end sizes of the NFRP element, the antenna was capable of switching its polarization state from RHCP to LHCP and vice versa. The reconfigurability is realized by controlling the ON/OFF states of 8 PIN-diodes, which are inserted into the arrowhead endings of the NFRP element. With the features of switchable polarization sense, compact size, good impedance matching, high radiation efficiency, the proposed antenna can be widely applied to the wireless communication systems with $\mathrm{CP}$ antenna characteristics.

\section{ACKNOWLEDGMENT}

This research is funded in part by Vietnam National Foundation for Science and Technology Development (NAFOSTED) under grant number 102.04-2016.02 and in part by Institute for Information \& Communications Technology Promotion (IITP) grant funded by the Korea government (MSIT) (No. 2017-0-00959, University ICT Basic Research Laboratory).

\section{REFERENCES}

[1] A. Khidre, K. Lee, F. Yang, and A. Elshebeni, "Circular polarization reconfigurable wideband E-shaped patch antenna for wireless applications," IEEE Trans. Antennas Propag., vol. 61, no. 2, pp. 960 964, Feb. 2013.

[2] N. Nguyen, L. Hall, and C. Fumeaux, "A frequency- and polarizationreconfigurable stub-loaded microstrip patch antenna," IEEE Trans. Antennas Propag., vol. 63, no. 11, pp. 5235-5240, Nov. 2015.

[3] H. Aissat, L. Cirio, M. Grzeskowiak, J. Laheurte, and O. Picon, "Reconfigurable circularly polarized antenna for short-range communication systems," IEEE Trans. Microw. Theory Techn., vol. 54, no. 6, pp. 2856-2863, Jun. 2006.

[4] R. Chen and J. Row, "Single-fed microstrip patch antenna with switchable polarization," IEEE Trans. Antennas Propag., vol. 56, no. 4, pp. 922-926, Apr. 2008.

[5] W. Yoon, S. Han, J. Baik, S. Pyo, J. Lee, and Y. Kim, "Crossed dipole antenna with switchable circular polarization sense," Electron. Lett., vol. 45, no. 14, pp. 717-718, Jul. 2009.

[6] W. Lin and H. Wong, "Wideband circular polarization reconfigurable antenna," IEEE Trans. Antennas Propag., vol. 63, no. 12, pp. 5938 5944, Dec. 2015.

[7] L. Zhang, S. Gao, Q. Luo, P. Young, and Q. Li, "Wideband loop antenna with electronically switchable circular polarization," IEEE Antennas Wireless Propag. Lett. to be published.

[8] H. Zhu, S. Cheung, X. Liu, and T. Yuk, "Design of polarization reconfigurable antenna using metasurface," IEEE Trans. Antennas Propag., vol. 62, no. 6, pp. 2891-2898, Jun. 2014.

[9] W. Yang, W. Che, H. Jin, W. Feng, and Q. Xue, “A polarizationreconfigurable dipole antenna using polarization rotation AMC structure," IEEE Trans. Antennas Propag., vol. 63, no. 12, pp. 53055315, Dec. 2015.

[10] R. W. Ziolkowski, P. Jin, and C. Lin, "Metamaterial-inspired engineering of antennas," IEEE Proc., vol. 99, no. 10, pp. 1720-1731, Oct. 2011.

[11] M. Tang and R. W. Ziolkowski, "A frequency-agile, ultralow-profile, complementary slit ring resonator-based electrically small antenna," Microwave Opt. Technol. Lett., vol. 55, no. 10, pp. 2425-2428, Oct. 2013. 
[12] Y. Yu, J. Xiong, H. Li, and S. He, "An electrically small frequency reconfigurable antenna with a wide tuning range," IEEE Antennas Wireless Propag. Lett., vol. 10, pp. 103-106, 2011.

[13] S. X. Ta, K. Lee, I. Park, and R. W. Ziolkowski, "Compact crosseddipole antenna loaded with near-field resonant parasitic element," IEEE Trans. Antennas Propag., vol. 65, no. 2, pp. 482-488, Feb. 2017.

[14] S. X. Ta, I. Park, and R. W. Ziolkowski, "Broadband electrically small circularly polarized directive antenna," IEEE Access, vol. 5, pp. 14657-14663, Jul. 2017.

[15] S. X. Ta, I. Park, and R. W. Ziolkowski, "Crossed dipole antennas: a review," IEEE Antennas Propag. Mag., vol. 57, no. 5, pp. 107-122, Oct. 2015.

[16] Data Sheet of MA4SPS402 PIN Diodes, MA-Com, Application Note. 